\title{
Transvaginal ultrasonography and hysteroscopy in postmenopausal bleeding a prospective study
}

\author{
Rita Sousa ${ }^{1}$, Margarida Silvestre ${ }^{1}$, Luis Almeida e Sousa ${ }^{1}$, Francisco Falcão ${ }^{1}$, Isabel Dias ${ }^{1}$, Teresa Silva ${ }^{2}$, \\ Carlos De Oliveira ${ }^{1}$ and Henrique Miguel Oliveira ${ }^{1}$ \\ From the Departments of ${ }^{1}$ Gynecology and ${ }^{2}$ Pathology; University Hospital of Coimbra, Coimbra, Portugal
}

\begin{abstract}
Acta Obstet Gynecol Scand 2001; 80: 856-862. (C) Acta Obstet Gynecol Scand 2001
Objective. To determine the diagnostic value of transvaginal ultrasonography and hysteroscopy in patients with postmenopausal bleeding.

Material and methods. Between January 1, 1998 and June 30, 1999, 88 outpatient women with postmenopausal bleeding were enrolled in a prospective study. They underwent transvaginal ultrasonography and hysteroscopy, and were submitted to directed biopsy during hysteroscopy. Findings were classified as normal endometrium, suggestive of atrophy, focal abnormality (benign or suspicious), and diffuse thickness (benign or suspicious). Data was compared with the final diagnosis, established by histological examination, as atrophy, benign pathology, atypical hyperplasia and endometrial carcinoma.

Results. Among 88 women enrolled, 15 were excluded because hysteroscopy was impossible, and four had abandoned the study. The histological findings were scanty material in 12 $(17.4 \%)$, atrophy in $24(34.8 \%)$, cystic atrophy in one $(1.4 \%)$, normal endometrium in five $(7.2 \%)$, tuberculous endometritis in one (1.4\%), polyps in $12(17.4 \%)$, leiomyoma in one $(1.4 \%)$, non-atypical hyperplasia in three $(4.3 \%)$, atypical hyperplasia in one $(1.4 \%)$ and endometrial carcinoma in nine cases $(13.0 \%)$. For the assessment of endometrial carcinoma, ultrasonography revealed sensitivity $77.8 \%$, specificity $93.3 \%$, positive predictive value $63.6 \%$, negative predictive value $96.6 \%$; and hysteroscopy revealed sensitivity of $88.9 \%$, specificity $98.3 \%$, positive predictive value $88.9 \%$, negative predictive value $98.3 \%$. The combined use of both methods revealed sensitivity $100 \%$, specificity $91.7 \%$, positive predictive value $64.3 \%$, negative predictive value $100 \%$.

Conclusions. Both imagiological methods were found to be useful screening tests for endometrial carcinoma. Hysteroscopy was a superior diagnostic procedure.
\end{abstract}

Key words: endometrial carcinoma; histology; hysteroscopy; postmenopausal bleeding; transvaginal ultrasonography

Submitted 19 October, 2000

Accepted 25 April, 2001

Postmenopausal bleeding is a common gynecologic complaint, accounting for up to $69 \%$ of postmenopausal women referred to gynecological outpatient clinics (1). Approximately $90 \%$ of women with endometrial carcinoma report vaginal bleeding as their only complaint $(2,4)$, so this

\section{Abbreviations:}

D\&C: dilatation and curettage; HRT: hormone replacement therapy; IU: international unit. symptom should always be carefully investigated. However, just $10-15 \%$ of women with postmenopausal bleeding have endometrial carcinoma $(2,3,5-8)$.

Dilatation and curettage (D\&C) is still considered the 'gold standard' for the investigation of postmenopausal bleeding (9), although it is an invasive procedure, requiring inpatient admission and general anesthesia, associated with some morbidity and even mortality $(5,9)$. As a blind pro- 
cedure, it often results in unrepresentative biopsies (8) with a diagnostic failure that varies from 10 to $25 \%$ (10), and false negative rates between 2 and $10 \%(1,5,9,11)$. Prior reports have revealed that in $60 \%$ of women submitted to curettage less than half of the uterine cavity was sampled with the curette and that the source of bleeding was frequently not diagnosed $(12,13)$.

Diagnostic hysteroscopy allows direct visualization and biopsy of diffuse or focal abnormalities of the endometrium. It is a well-tolerated, accurate, and sensitive outpatient procedure, and a high predictive value in the investigation of postmenopausal bleeding has been well documented (1, $6,8,10,11,13,14)$. It is, however, a rather invasive method and carries a false negative rate of 3\% (11).

Transvaginal ultrasonography with high-resolution transducers allows a good definition of the endometrial-myometrial interface and visualization of the entire endometrial cavity. Several studies have assessed the accuracy of transvaginal ultrasonography in evaluating the endometrium for malignancy $(8,11,15-18)$. Different cut-off levels for endometrial thickness have been used, resulting in varying degrees of reliability for detecting endometrial disease $(1,3,6,8,9,11,17$, 18). Transvaginal ultrasonography is a highly sensitive test for detecting endometrial disease, but carries a false negative rate of $8 \%$ for endometrial carcinoma (15). Recent reports showed that the combined assessment of endometrial thickness with some morphologic parameters improve the diagnostic accuracy of transvaginal sonography in patients with postmenopausal bleeding (5).

The purpose of our study was to determine the diagnostic accuracy of transvaginal ultrasonography and hysteroscopy in women with postmenopausal bleeding.

\section{Material and methods}

Between January 1, 1998 and June 30, 1999, 88 women with postmenopausal bleeding were enrolled in a prospective study at the Department of Gynecology of University Hospital of Coimbra. These patients were consecutively recruited from gynecologic outpatient clinics, emergency departments or directly from general practice for investigation of postmenopausal bleeding, according to a previously defined protocol. The patients first underwent a standard outpatient evaluation. Reproductive history and a complete medical and surgical history, giving special attention to hypertension, diabetes, obesity, use of hormone replacement therapy (HRT), anti-estrogen therapy, anticoagulants or other therapies were recorded. Menopause was defined as spontaneous cessation of menses for more than one year or serum folliclestimulating hormone levels $>30 \mathrm{mIU} / \mathrm{ml}$. A general physical examination and a careful gynecologic examination, including cervical smear were performed. Data was collected in a standard format for all patients. Each patient gave informed consent to participate in the study.

Ultrasonography was performed with the use of an Aloka SSD 2000 MultiView with a $5 \mathrm{MHz}$ transvaginal transducer by one of the authors (L.A.S.). Endometrial thickness was measured as a double layer in the longitudinal plane at the widest point within the fundus and with the entire endocervical and endometrial stripe visible. Uterine contour was evaluated and the presence of intramural or submucosal lesions, as well as adnexial pathology were recorded. The ultrasonographic criteria considered to define a normal endometrium were normal endometrial thickness $(\leq 4$ $\mathrm{mm}$ without HRT and $\leq 8 \mathrm{~mm}$ with HRT) and endometrial regularity. Endometrial ultrasonographic findings were classified as normal endometrium, suggestive of atrophy, focal abnormality (benign or suspicious) and diffuse thickness (benign or suspicious).

Hysteroscopy was performed with a $4 \mathrm{~mm}$ Storz II rigid hysteroscope and the uterine cavity was distended with a low-flow, high-pressure carbon dioxide insuflation system, without paracervical block nor cervical dilatation before the procedure. It was performed immediately after ultrasonography by either one of two authors (F.F., I.D.) who were not aware of the sonographic results. The images were viewed on a high-resolution color monitor. The hysteroscope was guided through the endocervical canal into the uterine cavity under visual control. The cavity and endometrial surface were inspected systematically, and the tubal ostia identified by rotation of the hysteroscope. Hysteroscopic findings were classified as normal endometrium, suggestive of atrophy, focal abnormality (benign or suspicious) and diffuse thickness (benign or suspicious).

Endometrial biopsy was performed at the end of hysteroscopy with a Novak curette or by an directed biopsy. Histologic diagnosis was given by an associate investigator (T.S.) who was blinded to the ultrasonographic and hysteroscopic findings, as atrophy, benign pathology (polyp, submucous leiomyoma or non-atypical hyperplasia), atypical hyperplasia, and endometrial carcinoma. Patients whose collected specimens were classified as scanty material were included in the simple atrophy group.

After completing the protocol examinations, women were re-evaluated in an outpatient clinic, where data was analyzed, and were submitted to 
Table I. Characteristics of the study group

\begin{tabular}{lc}
\hline & $\begin{array}{c}\text { Patients } \\
(n=69)\end{array}$ \\
\hline Age (years) & ${ }^{*} 62.1 \pm 8.9(43-82)$ \\
Years after menopause (years) & ${ }^{2} 12.7 \pm 9.6(1-38)$ \\
HRT & $13(18.8 \%)$ \\
Hypertension & $25(36.2 \%)$ \\
Diabetes mellitus & $8(11.6 \%)$ \\
Anticoagulant therapy & $6(8.7 \%)$ \\
Other therapies & $6(8.7 \%)$ \\
\hline
\end{tabular}

${ }^{\star}$ mean \pm s.d.

medical or surgical therapy if necessary. Histologic diagnoses were compared to the ultrasonographic and hysteroscopic findings, and the sensitivity, specificity positive predictive value, and negative predictive value were calculated.

All procedures followed the ethical guidelines approved by the authorities of our University Hospital.

\section{Results}

Among 88 women enrolled in the study, just 69 had completed the protocol: $15(17 \%)$ were excluded because of impracticable hysteroscopy due to cervical stenosis and the remaining four $(4.5 \%)$ patients abandoned the study. The mean age of women participating in the study was $62.1 \pm 8.9$ years, ranging from 43 to 82 years, and they were postmenopausal for an average of $12.7 \pm 9.6$ years, ranging from 1 to 38 years. Just 13 women (18.8\%) had ever had hormone replacement therapy. The characteristics of the study group are listed in Table I.

Transvaginal ultrasonography was performed in all women. Ultrasonographic findings were (Fig. 1)

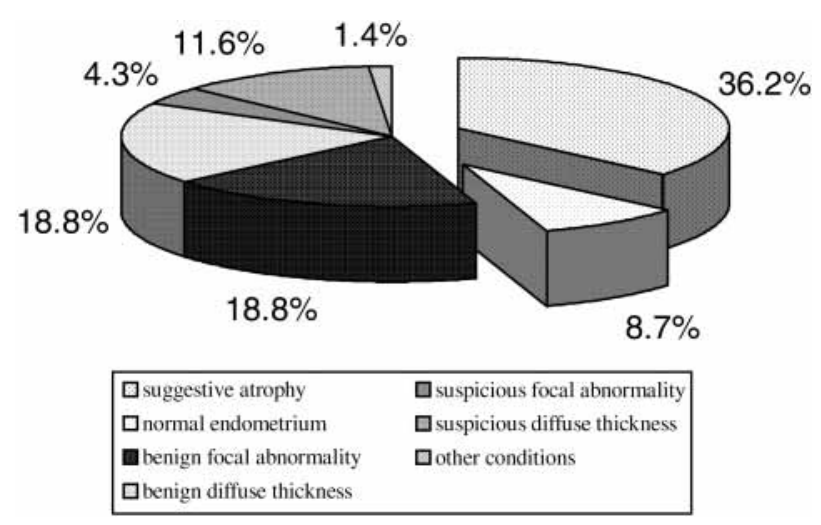

Fig. 1. Ultrasonographic findings.

normal endometrium in six $(8.7 \%)$, suggestive of atrophy in $25(36.2 \%)$, benign focal abnormality in $13(18.8 \%)$, suspicious focal abnormality in three (4.3\%), benign diffuse thickness in $13(18.8 \%)$, suspicious diffuse thickness in eight $(11.6 \%)$, and intracavitary fluid in one $(1.4 \%)$. Ten women $(14.5 \%)$ presented associated myometrial pathology (fibroids), and three patients $(4.3 \%)$, presented associated adnexial pathology (two simple ovarian cysts and one ovarian carcinoma). Mean endometrial thickness was $10.3 \pm 10.9 \mathrm{~mm}$ (min. 0 , max. 45 $\mathrm{mm})$, being $\leq 4 \mathrm{~mm}$ in $23(33.3 \%)$ cases, $4.1-8 \mathrm{~mm}$ in $18(26.1 \%), 8.1-12 \mathrm{~mm}$ in eight $(11.6 \%), 12.1-$ $16 \mathrm{~mm}$ in eight (11.6\%), 16.1-20 mm in two (2.9\%) and $>20 \mathrm{~mm}$ in ten $(14.5 \%)$. Table II shows the incidence of normal and pathological endometrial results in relation to endometrial thickness.

Hysteroscopy was impossible to perform in 15 women who were excluded from the protocol. Hysteroscopic findings were (Fig. 2) normal endometrium in two $(2.9 \%)$, suggestive of atrophy in 22

Table II. Incidence of normal and pathological endometrial results in relation to endometrial thickness

\begin{tabular}{|c|c|c|c|c|}
\hline \multirow[b]{2}{*}{ Endometrial thickness $(\mathrm{mm})$} & \multicolumn{4}{|c|}{$\begin{array}{l}\text { Histologic diagnosis } \\
\qquad(n=69)\end{array}$} \\
\hline & $\begin{array}{l}\text { Normal endometrium/Atrophy } \\
\qquad n=25\end{array}$ & $\begin{array}{c}\text { Benign pathology } \\
\quad n=34\end{array}$ & $\begin{array}{l}\text { Suspicious pathology } \\
\qquad n=1\end{array}$ & $\begin{array}{l}\text { Endometrial carcinoma } \\
\qquad n=9\end{array}$ \\
\hline$\leq 4$ & 16 & 7 & - & - \\
\hline$(n=23)$ & $(23.2 \%)$ & $(10.1 \%)$ & & \\
\hline $\begin{array}{l}4.1-8 \\
(n=18)\end{array}$ & $\begin{array}{c}9 \\
(13.0 \%)\end{array}$ & $\begin{array}{c}9 \\
(13.0 \%)\end{array}$ & - & - \\
\hline $\begin{array}{l}8.1-12 \\
(n=8)\end{array}$ & - & $\begin{array}{c}7 \\
(10.1 \%)\end{array}$ & - & $\begin{array}{c}1 \\
(1.4 \%)\end{array}$ \\
\hline $\begin{array}{l}12.1-16 \\
(n=8)\end{array}$ & - & $\begin{array}{c}4 \\
(5.8 \%)\end{array}$ & - & $\begin{array}{c}4 \\
(5.8 \%)\end{array}$ \\
\hline $\begin{array}{l}16.1-20 \\
(n=2)\end{array}$ & - & $\begin{array}{c}1 \\
(1.4 \%)\end{array}$ & $\begin{array}{c}1 \\
(1.4 \%)\end{array}$ & - \\
\hline $\begin{array}{l}>20 \\
(n=10)\end{array}$ & - & $\begin{array}{c}6 \\
(8.7 \%)\end{array}$ & - & $\begin{array}{c}4 \\
(5.8 \%)\end{array}$ \\
\hline
\end{tabular}



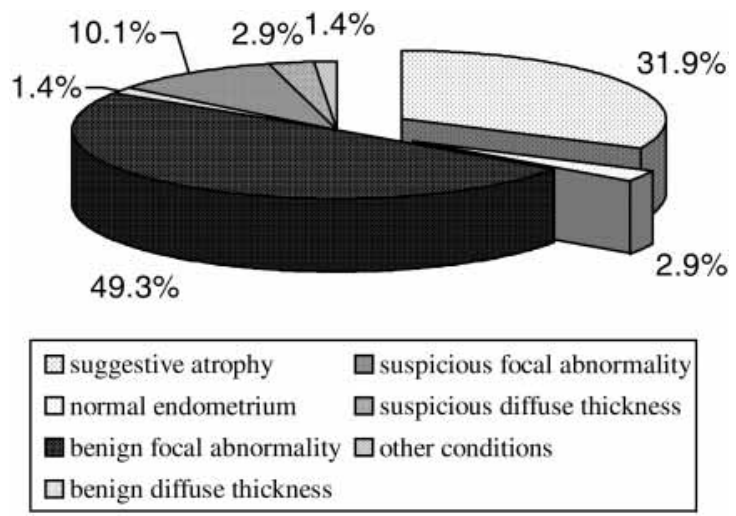

Fig. 2. Hysteroscopic findings.

(31.9\%), benign focal abnormality in $34(49.3 \%)$, suspicious focal abnormality in seven (10.1\%), benign diffuse thickness in one $(1.4 \%)$ and suspicious diffuse thickness in two $(2.9 \%)$. Purulent endometritis was found in one case (1.4\%).

The correlation between ultrasonographic and hysteroscopic findings are shown in Table III. The main differences concerned the discrimination between diffuse and focal abnormalities. Ten cases classified as benign diffuse thickness at ultrasonography were found to be benign focal abnormalities at hysteroscopy.

Endometrial sampling was performed by directed biopsy in $14(20.3 \%)$ women and by Novak curettage in the others. Endometrial pathology was revealed in 27 women $(39.1 \%)$. Histologic findings consisted of (Fig. 3) scanty material in 12 $(17.4 \%)$ cases, normal endometrium in five $(7.2 \%)$, atrophy in $24(34.8 \%)$ cases, cystic atrophy in one $(1.4 \%)$ case, tuberculous endometritis in one $(1.4 \%)$, polyps in $12(17.4 \%)$ cases, leiomyoma in one $(1.4 \%)$ case, non-atypical hyperplasia in three $(4.3 \%)$ cases, atypical hyperplasia in one $(1.4 \%)$ case and endometrial carcinoma in nine cases $(13.0 \%)$.

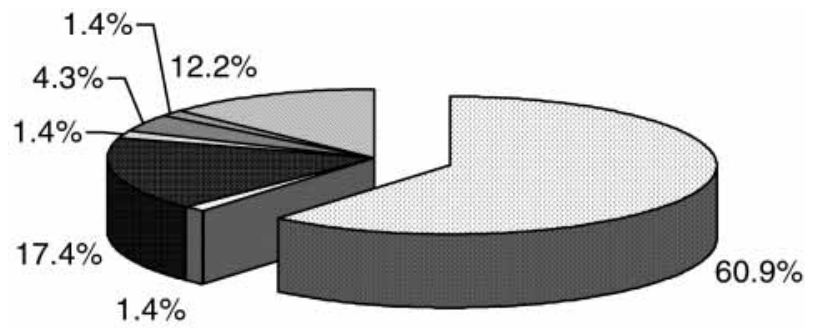

\begin{tabular}{|ll|}
\hline$\square$ atrophy/scanty material/normal endometrium & $\square$ non-atypical hyperplasia \\
$\square$ endometritis & $\square$ atypical hyperplasia \\
$\mathbf{\square}$ polyps & $\square$ endometrial carcinoma \\
$\square$ leiomyoma & \\
\hline
\end{tabular}

Fig. 3. Pathologic findings.

Thirty-nine women had surgical therapy: 22 women were submitted to operative hysteroscopy with resection of 21 polyps and 1 leiomyoma, and 17 women were submitted to laparotomy with total hysterectomy and bilateral adnexectomy (eight for endometrial carcinoma, one for atypical hyperplasia, four for benign pathology and two for associated adnexial pathology). The final diagnosis established by histology and the comparison of this diagnosis with biopsies is listed in Table IV. The discrepancy between biopsy results and operative histologic results mainly concerned focal endometrial abnormalities (in nine polyps and one leiomyoma the biopsy result was atrophy/scanty material).

Table V shows the relationship between ultrasonographic and hysteroscopic findings and histologic results obtained either by biopsy or surgical procedure.

There were $13(18.8 \%)$ women who were submitted to hormone replacement therapy (HRT), using a combined estrogen-progestin regimen. Their mean age was 55.5 \pm 5.6 years $(\min .50$, max. 70 years) and were postmenopausal for $6.7 \pm 4.8$ years (min. 1, max. 18 years). In this group of patients,

\begin{tabular}{|c|c|c|c|c|c|c|c|c|}
\hline & & & & & $\begin{array}{l}\text { Ultrasonogr } \\
\qquad(n=69)\end{array}$ & & & \\
\hline & & $\begin{array}{l}\text { Suggestive } \\
\text { atrophy }\end{array}$ & $\begin{array}{c}\text { Normal } \\
\text { endometrium }\end{array}$ & $\begin{array}{l}\text { Benign focal } \\
\text { abnormality }\end{array}$ & $\begin{array}{c}\text { Benign } \\
\text { diffuse } \\
\text { thickness }\end{array}$ & $\begin{array}{l}\text { Suspicious } \\
\text { focal } \\
\text { abnorm. }\end{array}$ & $\begin{array}{c}\text { Suspicious } \\
\text { diffuse thickness }\end{array}$ & $\begin{array}{c}\text { Other } \\
\text { conditions }\end{array}$ \\
\hline ஓ & Suggestive atrophy & 19 & 2 & 1 & - & - & - & - \\
\hline$\stackrel{\|}{=}$ & Normal endometrium & 1 & - & - & 1 & - & - & - \\
\hline$=$ & Benign focal abnormality & 5 & 4 & 11 & 10 & 2 & 2 & - \\
\hline : & Benign diffuse thickness & - & - & - & - & - & 3 & - \\
\hline లై & Suspicious focal abnorm. & - & - & 1 & 2 & 1 & 2 & - \\
\hline$\frac{\Phi}{\omega}$ & Suspicious diffuse thickness & - & - & - & - & - & 2 & - \\
\hline 齐 & Other conditions & - & - & - & - & - & - & $1^{*}$ \\
\hline
\end{tabular}

* Purulent (tuberculous) endometritis. 
Table IV. Endometrial biopsy results versus final histologic diagnosis in women submitted to operative hysteroscopy or hysterectomy at the end of the study

\begin{tabular}{|c|c|c|c|c|c|c|c|c|c|}
\hline & \multicolumn{9}{|c|}{$\begin{array}{l}\text { Endometrial biopsy results } \\
\qquad(n=39)\end{array}$} \\
\hline & & $\begin{array}{c}\text { Atrophy } \\
\text { /scanty material }\end{array}$ & $\begin{array}{c}\text { Normal } \\
\text { endometrium }\end{array}$ & Polyp & Leiomyoma & $\begin{array}{l}\text { Non-atypical } \\
\text { hyperplasia }\end{array}$ & $\begin{array}{c}\text { Atypical } \\
\text { hyperplasia }\end{array}$ & $\begin{array}{l}\text { Endometrial } \\
\text { carcinoma }\end{array}$ & $\begin{array}{l}\text { Other } \\
\text { conditions }\end{array}$ \\
\hline \multirow{8}{*}{ 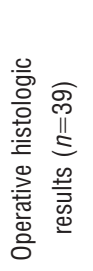 } & Atrophy/scanty material & & & & \multirow{8}{*}{1} & \multirow{8}{*}{2} & \multirow{8}{*}{1} & \multirow{8}{*}{8} & \\
\hline & Normal endometrium & & & & & & & & \\
\hline & Polyp & 9 & 2 & 10 & & & & & \\
\hline & Leiomyoma & 1 & & & & & & & \\
\hline & Non-atypical hyperplasia & & & & & & & & \\
\hline & Atypical hyperplasia & & & & & & & & \\
\hline & Endometrial carcinoma & & & & & & & & \\
\hline & Other conditions & 3 & & 2 & & & & & \\
\hline
\end{tabular}

Table V. Relationship between ultrasonographic and hysteroscopic findings and histologic results obtained either by biopsy or surgical procedure

\begin{tabular}{|c|c|c|c|c|c|c|c|c|c|}
\hline & \multicolumn{9}{|c|}{$\begin{array}{l}\text { Histologic diagnosis } \\
\qquad(n=69)\end{array}$} \\
\hline & & $\begin{array}{c}\text { Atrophy } \\
\text { /scanty material }\end{array}$ & $\begin{array}{l}\text { Normal } \\
\text { endometrium }\end{array}$ & Polyp & Leiomyoma & $\begin{array}{c}\text { Non-atypical } \\
\text { hyperplasia }\end{array}$ & $\begin{array}{c}\text { Atypical } \\
\text { hyperplasia }\end{array}$ & $\begin{array}{c}\text { Endometrial } \\
\text { carcinoma }\end{array}$ & $\begin{array}{c}\text { Other } \\
\text { conditions }\end{array}$ \\
\hline \multirow{7}{*}{ 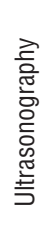 } & Suggestive atrophy & 19 & 1 & 3 & 2 & & & & \\
\hline & Normal endometrium & 1 & 1 & 2 & & & & & $2^{*}$ \\
\hline & Benign focal abnormality & 1 & & 10 & 1 & & & 1 & \\
\hline & Benign diffuse thickness & & 2 & 7 & & 1 & 1 & 1 & $1^{*}$ \\
\hline & Suspicious focal abnorm. & & & 2 & & & & 1 & \\
\hline & Suspicious diffuse thick. & & & 1 & & & & 6 & $1^{*}$ \\
\hline & Other conditions & & & & & & & & $1^{\ddagger}$ \\
\hline \multirow{7}{*}{ 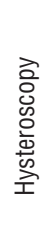 } & Suggestive atrophy & 19 & 2 & 1 & & & & & \\
\hline & Normal endometrium & 1 & 1 & & & & & & \\
\hline & Benign focal abnormality & 1 & 1 & 24 & 3 & 1 & & 1 & $3^{*}$ \\
\hline & Benign diffuse thickness & & & & & & & & $1^{*}$ \\
\hline & Suspicious focal abnorm. & & & & & & 1 & 6 & \\
\hline & Suspicious diffuse thick. & & & & & & & 2 & \\
\hline & Other conditions & & & & & & & & $1^{\ddagger}$ \\
\hline
\end{tabular}

‡ tuberculous endometritis; * cystic atrophy.

the mean endometrial thickness measured by transvaginal ultrasonography was $5.7 \pm 3.1 \mathrm{~mm}$ (ranging from 1 to $11 \mathrm{~mm}$ ), and the ultrasonographic findings were normal endometrium in three, suggestive of atrophy in six, benign focal abnormality in two, and benign diffuse thickness in two patients. Hysteroscopy revealed normal endometrium in one, endometrium suggestive of atrophy in seven, and benign focal abnormality in five patients. In nine patients endometrial pathology was not observed (atrophy in seven, normal endometrium in two), and the remaining four patients presented benign endometrial pathology (polyps in three and non-atypical hyperplasia in one).

Endometrial carcinoma was detected in nine $(13.0 \%)$ women. Their mean age was $69.4 \pm 10.8$ years, ranging from 52 to 82 years and they were postmenopausal for $20.2 \pm 13.0$ years, ranging from one to 38 years. Among this group none of the patients used HRT; five patients suffered from hypertension and two patients were diabetic. The mean endometrial thickness measured by transvaginal ultrasonography was $21.4 \pm 9.5 \mathrm{~mm}$ (min. 10 $\mathrm{mm}$, max. $38 \mathrm{~mm}$ ). Ultrasonographic findings were suspicious diffuse thickness in six, suspicious focal abnormality in one, benign focal abnormality in one, and benign diffuse thickness in one patient. Hysteroscopic findings were suspicious diffuse thickness in two, suspicious focal abnormality in six, and benign focal abnormality in one patient.

For assessment of endometrial carcinoma, transvaginal ultrasonography revealed sensitivity $77.8 \%$, specificity $93.3 \%$, positive predictive value $63.6 \%$, negative predictive value $96.6 \%$; hysteroscopy revealed sensitivity $88.9 \%$, specificity $98.3 \%$, positive predictive value $88.9 \%$, negative predictive 
value $98.3 \%$. Ultrasonography, with evaluation of endometrial thickness alone, using a cut-of value of $4 \mathrm{~mm}$, showed a sensitivity, specificity, positive predictive value and negative predictive value for detecting endometrial carcinoma of $100 \%, 46 \%$, $19.6 \%$ and $100 \%$, respectively. The combined use of ultrasonography and hysteroscopy revealed sensitivity $100 \%$, specificity $91.7 \%$, positive predictive value $64.3 \%$, negative predictive value $100 \%$.

For the assessment of endometrial pathology as a whole, ultrasonography revealed sensitivity $79.5 \%$, specificity $88 \%$, positive predictive value $92.1 \%$, negative predictive value $71 \%$; and hysteroscopy revealed sensitivity $97.7 \%$, specificity $92 \%$, positive predictive value $95.5 \%$, negative predictive value $95.8 \%$. The combined use of ultrasonography and hysteroscopy revealed sensitivity $97.7 \%$, specificity $84.0 \%$, positive predictive value $91.5 \%$, negative predictive value $95.5 \%$.

\section{Discussion}

Postmenopausal bleeding is the presenting complaint in approximately $90 \%$ of patients with endometrial carcinoma (2). However, endometrial carcinoma contributes to $10-15 \%$ of postmenopausal bleeding and the majority of patients with this bleeding have no detectable organic cause $(4,17)$. Dilatation and curettage (D\&C) still remains the usual diagnostic procedure for these patients, despite inherent diagnostic limitations and the fact that it is an invasive inpatient procedure $(5,9)$. Furthermore, focal benign abnormalities are usually missed by this procedure and may be a source of continued or recurrent bleeding (2).

In the present study we have detected endometrial pathology in $63.8 \%$ of women with postmenopausal bleeding, which is slightly superior to previous reports $(2,14,15)$. Moreover, in our study, endometrial carcinoma had a prevalence of $13 \%$. Furthermore, combining transvaginal ultrasonography and hysteroscopy, which represented our methodology, not a single endometrial carcinoma was missed.

Transvaginal ultrasonography was performed in all patients and was well tolerated. Findings were classified according to endometrial thickness and, using morphological criteria, in six classes (normal endometrium, suggestive of atrophy, benign focal abnormality, suspicious focal abnormality, benign diffuse thickness and suspicious diffuse thickness). According to this classification, ultrasonography revealed a sensitivity, specificity, positive predictive value and negative predictive value of $79.5 \%, 88 \%$, $92.1 \%$ and $71 \%$, respectively, referring to endometrial pathology. Comparing these results with previous reports, we found a lower sensitivity $(79.5 \%$ vs. $97 \%$ as obtained by Weber et al. (5), who combined endometrial morphology, endometrial border and endometrial thickness, and used a cut-off value of $5 \mathrm{~mm}$ ), nevertheless a higher specificity $(88 \%$ vs. 65\%) (5) was observed in our study. The low sensitivity of transvaginal ultrasonography observed in our study resulted from the fact that this technique missed seven cases of benign pathology with endometrial thickness inferior to $4 \mathrm{~mm}$ (five endometrial polyps measuring less than $3 \mathrm{~mm}$ and two small submucous leiomyomas covered by thin endometrium).

Considering endometrial carcinoma, transvaginal ultrasonography parameters revealed values of sensitivity $77.8 \%$, specificity $93.3 \%$, positive predictive value $63.6 \%$, and negative predictive value $96.6 \%$. If endometrial thickness was the only parameter considered, using a cut-off value of $4 \mathrm{~mm}$ for malignant pathology, ultrasonography revealed a sensitivity of $100 \%$ and negative predictive value of $100 \%$, but a specificity of $46 \%$ and positive predictive value of $19.6 \%$. Actually we would have obtained the same results using a cut-off value of $8 \mathrm{~mm}$, because, in our series, no endometrial carcinoma was observed below the cut-off value of $10 \mathrm{~mm}$. These values are comparable to previous reports that showed a high sensitivity of transvaginal ultrasonographic measurement of endometrial thickness in excluding endometrial carcinoma, using cut-off values of $4-5 \mathrm{~mm}(3,4,6,9,14,15,17)$. These reports conclude that endometrial carcinoma is an uncommon finding among women with a thin endometrium in transvaginal utrasonography $(11,15)$. The use of transvaginal ultrasonographic morphologic parameters to assess endometrial pathology reveals a lower sensitivity but higher specificity and increased negative predictive value for malignant pathology.

Hysteroscopy gives evidence of a high diagnostic accuracy, with sensitivity $97.7 \%$, specificity $92 \%$, positive predictive value $95.5 \%$ and negative predictive value $95.8 \%$ for endometrial pathology, according to previous reports $(1,6,8,10,11,13,14)$. Direct visualization of the uterine cavity is a simple and effective method for investigating endometrial and even endocervical pathologies (6). Hysteroscopy showed great efficiency in the diagnosis of focal abnormalities of the endometrium $(4,11)$, which are hardly recognized by ultrasonography. Hysteroscopy was also demonstrated to be of great value to delineate an adequate therapeutic management for benign intracavitary pathology. However, it is a rather invasive procedure, so that, in our study, among 15 women (17\%), hysteroscopy was impossible as an outpatient procedure, because of cervical stenosis. 
For assessment of endometrial carcinoma, hysteroscopy revealed sensitivity of $88.9 \%$, specificity of $98.3 \%$, positive predictive value of $88.9 \%$ and negative predictive value of $98.3 \%$. There was one endometrial carcinoma initially classified as benign focal abnormality, but directed biopsy established the correct diagnosis.

The combined use of ultrasonography and hysteroscopy, associated with endometrial sampling, did not miss any endometrial abnormality, namely endometrial carcinoma.

In conclusion, our results showed that ultrasonography is an accurate method for detection of endometrial pathology, and should be used as a first line technique in the assessment of postmenopausal bleeding. If the endometrial thickness measured by transvaginal ultrasonography is $\leq 4$ $\mathrm{mm}$, there is no need to perform hysteroscopy and biopsy, unless there are risk factors for endometrial carcinoma or recurrent bleeding.

Hysteroscopy was a superior diagnostic procedure and should be performed whenever an endometrial thickness is greater than $4 \mathrm{~mm}$, or illdefined endometrial lining, recurrent bleeding or risk factors for endometrial carcinoma are present.

\section{References}

1. Tahir MM, Bigrigg MA, Browning JJ, Brookes ST, Smith PA. A randomised controlled trial comparing transvaginal ultrasound, outpatient hysteroscopy and endometrial biopsy with inpatient hysteroscopy and curettage. Br J Obstet Gynaecol 1999; 106: 1259-64.

2. Lurain JR. Uterine cancer. In: Berek JS, Adashi EY, Hillard PA (eds.): Novak's Gynecology. 12th ed. Baltimore: Williams \& Wilkins, 1996: 1057-110.

3. Bakour SH, Dwarakanath LS, Kahn KS, Newton JR, Gupta JK. The diagnostic accuracy of ultrasound scan in predicting endometrial hyperplasia and cancer in postmenopausal bleeding. Acta Obstet Gynecol Scand 1999; 78: 447-51.

4. Giusa-Chiferi MG, Gonçalves WJ, Bacarat EC, Albuquerque Neto LC, Bortoletto CC, Lima GR. Transvaginal ultrasound, uterine biopsy and hysteroscopy for postmenopausal bleeding. Int J Gynaecol Obstet 1996; 55: 39 44.

5. Weber G, Merz E, Bahlmann F, Rösch B. Evaluation of different transvaginal parameters in women with postmenopausal bleeding. Ultrasound Obstet Gynecol 1998; 12: 265-70.
6. Haller H, Matejcic N, Rukavina B, Krasevic M, Rupcic S, Mozetic D. Transvaginal sonography and hysteroscopy in women with postmenopausal bleeding. Int J Gynaecol Obstet 1996; 54: 155-9.

7. O'Connor LP, Fries MH, Zeringue E, Brehm W. Triage of abnormal postmenopausal bleeding: a comparison of endometrial biopsy and transvaginal sonohysterography versus fractional curettage with hysteroscopy. Am J Obstet Gynecol 1998; 178: 956-61.

8. Loverro G, Bettochi S, Cormio G, Nicolardi V, Greco P, Vimercati A et al. Transvaginal sonography and hysteroscopy in postmenopausal uterine bleeding. Maturitas 1999; 33: $139-44$.

9. Büyük E, Durmusoglu F, Erenus M, Karakoç B. Endometrial disease diagnosed by transvaginal ultrasound and dilatation and curettage. Acta Obstet Gynecol Scand 1999; 78: 419-22.

10. Nagele F, O'Connor H, Baskett T, Davies A, Mohammed $\mathrm{H}$, Magos AL. Hysteroscopy in women with uterine bleeding on hormone replacement therapy: a comparison with postmenopausal bleeding. Fertil Steril 1996; 65: 114550 .

11. Gull B, Carlsson S, Karlsson B, Ylöstalo P, Milsom I, Granberg S. Transvaginal ultrasonography of the endometrium in women with postmenopausal bleeding: is it always necessary to perform an endometrial biopsy? Am J Obstet Gynecol 2000; 182: 509-15.

12. Stock RJ, Kanbur A. Prehysterectomy curettage. Obstet Gynecol 1975; 45: 537-41.

13. Towbin NA, Gviazda IM, March CM. Office hysteroscopy versus transvaginal ultrasonography in the evaluation of patients with excessive uterine bleeding. Am J Obstet Gynecol 1996; 174: 1678-82.

14. Garuti G, Sambruni I, Cellani F, Garzia D, Alleva P, Luerti M. Hysteroscopy and transvaginal ultrasonography in postmenopausal women with uterine bleeding. Int J Gynaecol Obstet 1999; 65: 25-33.

15. Smith-Bindman R, Kerlikowske K, Feldstein VA et al. Endovaginal ultrasound to exclude endometrial cancer and other endometrial abnormalities. JAMA 1998; 280: 151017.

16. Holbert TR. Transvaginal ultrasonographic measurement of endometrial thickness in postmenopausal women receiving estrogen replacement therapy. Am J Obstet Gynecol 1997; 176: 1334-9.

17. Briley M, Lindsell DRM. The role of transvaginal ultrasound in the investigation of women with post-menopausal bleeding. Clin Radiol 1998; 53: 502-5.

\section{Address for correspondence:}

Rita Sousa, M.D.

Serviço de Ginecologia, Hospitais da Universidade de Coimbra Praceta Prof. Mota Pinto

3049 Coimbra-Codex

Portugal 